\title{
Modeling the Pre-Breakdown V-I Characteristics of an Electrostatic Precipitator
}

\author{
B. S. Rajanikanth and D. V. S. Sarma \\ Department of High Voltage Engineering \\ Indian Institute of Science \\ Bangalore 560012, India
}

\begin{abstract}
A novel mathematical model for determining the electrical characteristics in a dc energized duct type electrostatic precipitator is described. The method is a quasianalytical one, based on solving the current continuity equation by finite-difference method and Poisson's equation by variational principle with the help of Rvachev functions (R-functions). The methodology described represents a valuable design tool for simulating and comparing the voltage-current characteristics of different wire-plate precipitator configurations before optimizing the geometric parameters namely shape of the corona wire, shape of the collection electrodes, wire cross-section, wire-wire and wire-plate spacing. The proposed method will be useful in trying innovative ideas in the design aspect of a wire-duct precipitator. Other significant features of this method are reduced problem domain, less memory space, and faster convergence. The proposed method has been validated with published experimental results and the agreement is excellent. A comparison of electrical characteristics has been made for different sizes and shapes of corona wire and also for various configurations of the wire-plate precipitators.
\end{abstract}

\section{INTRODUCTION}

$\mathrm{A}^{\mathrm{I}}$ IR pollution is one of the worst factors that spoil the environment and threaten life on this planet. Industrial emission (from power plants, cement plants etc.) is one of the major contributors to this pollution. Amongst existing pollution control devices, the electrostatic precipitator (ESP) is recognized to be eminently suitable for trapping fine particles of pollutants in large magnitudes. Since the precipitator will be in operation throughout the year its continuous online diagnosis and maintenance is very much needed to optimize its performance.

The Voltage-Current (V-I) characteristics are one of the major diagnostic tools being frequently used to monitor an ESP's performance. In the diagnosis, the dust free V-I characteristics are considered as the reference. Much work has been done to experimentally determine the dust free V-I characteristics while the ESP is in operation but at the expense of time and money. 1970's saw the development of computational techniques to predict the V-I characteristics. Since the same results can be obtained through simulations there is no need to resort to experiments, which usually are cumbersome and time-consuming. McDonald et al [1] developed the first ever numerical technique based on finite difference method (FDM), but the method proved to be highly time-consuming for larger grid sizes. In the next two decades, several innovative techniques and approaches were contributed to this field with the aim of reducing the computation time and memory. Medlin [2] made a brief survey of several existing methods. Recently Barbarics et al [3] used a combination of methods of characteristics and Rvachev (R) functions for field calculations inside an ESP. However, they have not checked the feasibility of their method for simulation of corona current characteristics.

Unlike the conventional numerical approach this $\mathrm{R}$ function technique is a semi-analytical technique but equally efficient in modeling any pattern of the corona and ground electrodes, which was rather difficult to model in the conventional approaches. In the proposed quasianalytical method, we have used for the first time, a combination of FDM and R-functions for modeling the dust free V-I characteristics thus testing the feasibility of the proposed method. Only one-quarter section has been used as the problem domain, which results in reduced memory and computation time. The possibility of modeling the corona wire and the grounded plate in any practically viable shape is the main advantage of the proposed method, which makes this method a design tool for novel ESP configurations. The results were validated against published experimental data and closer predictions have been achieved, when compared to other techniques. 


\section{MATHEMATICAL FORMULATION OF THE MODEL}

\subsection{NOMENCLATURE}

$\varphi_{0} \quad$ applied voltage $(\mathrm{V})$

$\varphi$ potential at any point $(\mathrm{V})$

$j$ current density $\left(\mathrm{A} / \mathrm{m}^{2}\right)$

$S_{x}$ wire-plate spacing (m)

$S_{y}$ one-half wire-to-wire spacing $(\mathrm{m})$

$j_{m}$ measured average current density at the plate $\left(\mathrm{A} / \mathrm{m}^{2}\right)$

$b$ mobility of charge carriers $\left(\mathrm{m}^{2} / \mathrm{V} \mathrm{s}\right)$

$a$ radius of the corona wire $(\mathrm{m})$

$D$ electric displacement $\left(\mathrm{A} \mathrm{s} / \mathrm{m}^{2}\right)$

$E$ electric field strength $(\mathrm{V} / \mathrm{m})$

$E_{\mathrm{x}}$ electric field in X-direction $(\mathrm{V} / \mathrm{m})$

$E_{\mathrm{y}}$ electric field in Y-direction $(\mathrm{V} / \mathrm{m})$

$E_{\mathrm{c}}$ electric field at corona wire $(\mathrm{V} / \mathrm{m})$

$\rho$ space-charge density $\left(\mathrm{C} / \mathrm{m}^{3}\right)$

$j_{\text {ave }}$ average current density at plate $\left(\mathrm{A} / \mathrm{m}^{2}\right)$

$\epsilon_{0}$ permittivity of air $(\mathrm{F} / \mathrm{m})$

$f$ roughness factor

$\delta$ relative air density factor

$a_{x}$ distance between two grid points in X-direction $(\mathrm{m})$

$a_{y}$ distance between two grid points in Y-direction $(\mathrm{m})$

$s$ thickness of wire of square cross-section (m)

\subsubsection{LIST OF SYMBOLS}

$\checkmark$ conjunction of functions

$\wedge$ disjunction of functions

$\cup$ union of regions

$\cap$ intersection of regions

\subsection{GOVERNING EQUATIONS}

The equations governing the electric field under steady-state conditions (in the absence of magnetic effects) for an Electrostatic Precipitator are

$$
\begin{gathered}
\quad \nabla \cdot D=\rho \\
\text { and } \quad \nabla \cdot j=0 \\
\text { where } \quad D=\varepsilon_{0} E \\
E=-\nabla \varphi \\
\text { and } j=\rho b E
\end{gathered}
$$

Equations (1), (3) and (4) yield the Poisson's equation,

$$
\nabla^{2} \varphi=\frac{-\rho}{\epsilon_{0}}
$$

In order to determine the electrical characteristics of a wire-duct precipitator it is necessary to solve current continuity Equation (2) and Poisson's Equation (6) simultaneously, subject to the existing boundary conditions.

\subsubsection{ASSUMPTIONS}

(i) The field on the wire is equal to the corona onset gradient given by Peek's formula [4].

(ii) The mobility of the charge carriers is uniform.

(iii) Thermal diffusion of ions is neglected.

(iv) The surface of the corona electrode is smooth.

\subsubsection{PROBLEM DOMAIN}

The nomenclature and coordinate system used are similar to those used in [1]. The problem is assumed to be two dimensional, since for clean and smooth wires there is no variation of voltage or current along the length of the corona wires. By using symmetry conditions, the computational domain over which the equations must be evaluated are reduced to only one-quarter section as shown in the Figure 1 (a). The Dirichlet and Neumann boundaries considered in the problem domain are as shown in the Figure 1 (b).

(a)

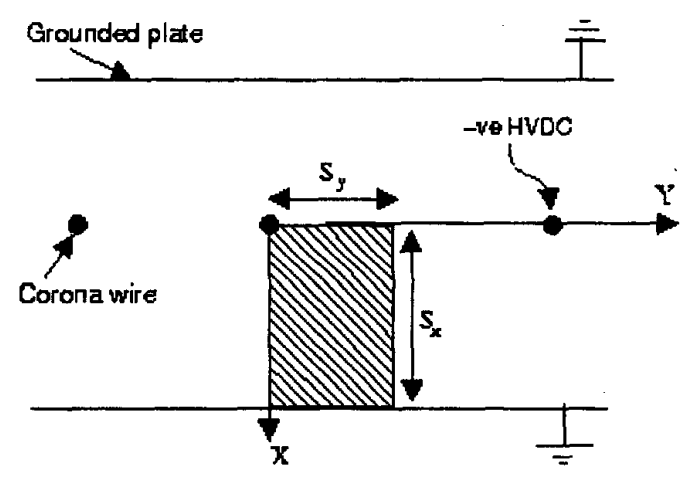

(b)

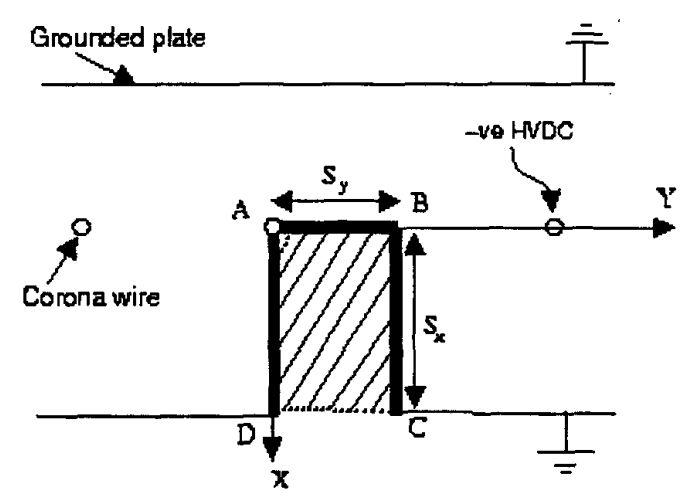

Figure 1. Problem domain in the ESP.

(a) Computational domain

(b) Boundary conditions 


\subsubsection{BOUNDARY CONDITIONS}

(i) The field on wire is equal to the corona onset gradient given by Peek's formula [4] as,

$$
E_{c}=3.1 \times 10^{6} \delta f\left(1+3.08 \times 10^{-2} /(\delta a)^{0.5}\right)
$$

(ii) The Dirichlet - type boundary conditions on the corona wire (point $\mathrm{A}$ in the Figure $1 \mathrm{~b}$ ) and the grounded plate (CD in the Figure $1 \mathrm{~b}$ ) have been satisfied as,

$$
\begin{aligned}
\left.\varphi\right|_{A} & =\varphi_{0} \\
\left.\varphi\right|_{D C} & =0
\end{aligned}
$$

where ' $A$ ' represents the high voltage electrode with applied potential $\varphi_{0}$ and 'DC' represents the grounded electrode.

(iii) The Neumann - type boundary conditions along ' $A B$ ', ' $B C$ ' and ' $A D$ ' have been satisfied as,

$$
\left.\frac{\partial \varphi}{\partial x}\right|_{A B}=0,\left.\frac{\partial \varphi}{\partial y}\right|_{B C}=0,\left.\frac{\partial \varphi}{\partial y}\right|_{A D}=0
$$

\subsection{SOLUTION OF THE CURRENT CONTINUITY EQUATION USING FDM}

The current continuity equation can be transformed into an equation involving potential $(\varphi)$ and space charge density $(\rho)$ by using Equations (2) and (5) as,

$$
\begin{gathered}
\nabla \cdot(\rho b E)=0 \\
\rho b(\nabla \cdot E)+b(E \cdot \nabla \rho)+\rho(E \cdot \nabla b)=0
\end{gathered}
$$

The third term in the Equation (11) becomes zero as the mobility of the charge carriers is assumed to be constant which results in,

$$
\rho b(\nabla \cdot E)+b(E \cdot \nabla \rho)=0
$$

On expanding the Equation (12) using the Equation (1) results in,

$$
\rho b\left(\frac{\rho}{\epsilon_{0}}\right)+b\left(E_{x} \frac{\partial \rho}{\partial x}+E_{y} \frac{\partial \rho}{\partial y}\right)=0
$$

At any point $(i, j)$ in the computational domain Equation (13) becomes,

$$
\left(\frac{\rho^{2}}{\epsilon_{0}}+E_{x} \frac{\partial \rho}{\partial x}+E_{y} \frac{\partial \rho}{\partial y}\right)_{(i, j)}=0
$$

The expressions for $(\partial \rho / \partial x)$ and $(\partial \rho / \partial y)$ are obtained from the backward difference method as,

$$
\begin{aligned}
& \frac{\partial \rho_{i, j}}{\partial x}=\frac{\rho_{i, j}-\rho_{i-1, j}}{a_{x}} \\
& \frac{\partial \rho_{i, j}}{\partial y}=\frac{\rho_{i, j}-\rho_{i, j-1}}{a_{y}}
\end{aligned}
$$

By substituting the expressions for $(\partial \rho / \partial x)$ and $(\partial \rho / \partial y)$ Equation (14) becomes a quadratic equation in $\rho$ as,

$$
\begin{aligned}
\left(\frac{a_{x} a_{y}}{\epsilon_{0}}\right) \rho_{i, j}^{2}+\left(a_{y} E_{x}+a_{x} E_{y}\right) \rho_{i, j} \\
-\left(a_{y} E_{x} \rho_{i-1, j}+a_{x} E_{y} \rho_{i, j-1}\right)=0
\end{aligned}
$$

By solving the Equation (17), the space charge density at any point $(i, j)$ on the grid can be computed. Central difference method has been used for calculating $E_{x}$ and $E_{y}[5]$ as,

$$
\begin{aligned}
& E_{x_{(i, j)}}=-\frac{\partial \varphi_{(i, j)}}{\partial x}=\frac{1}{2 a_{x}}\left(\varphi_{i+1, j}-\varphi_{i-1, j}\right) \\
& E_{y_{(i, j)}}=-\frac{\partial \varphi_{(i, j)}}{\partial y}=\frac{1}{2 a_{y}}\left(\varphi_{i, j+1}-\varphi_{i, j-1}\right)
\end{aligned}
$$

Initially the potential at any point $(i, j)$ in the grid has been calculated using Cooperman's analytical equation [1].

\subsection{SOLUTION OF THE POISSON'S EQUATION USING VARIATIONAL PRINCIPLE}

For stable configuration of the electromagnetic field the energy stored in the volume must have a minimum value. This minimum value principle is mathematically equivalent to the solution of the differential equation [6,7]. The energy related functional of the static electric field in a bounded region could be formulated with the scalar potential $\varphi$ as,

$$
W(\varphi)=\frac{1}{2} \int_{\Omega}\left(\epsilon_{0} \nabla \varphi \cdot \nabla \varphi-2 \rho \varphi\right) d \Omega
$$

The scalar potential function $(\varphi)$ is expressed as the sum of the known component $\left(\varphi_{\delta}\right)$ and unknown component $\left(\varphi_{\alpha}\right)$ as,

$$
\varphi=\varphi_{\delta}+\varphi_{\alpha}
$$

As Neumann-type boundaries are also considered in describing the problem domain an additional term is to be added to the Equation (21) and so potential function $(\varphi)$ becomes,

$$
\begin{gathered}
\varphi=\varphi_{\delta}+\varphi_{\alpha}+\varphi_{N} w_{D N} \\
\text { where } \quad \varphi_{N}=\nabla\left(\varphi_{\delta}+\varphi_{\alpha}\right) \cdot n \\
n=-\nabla\left(w_{N}\right)
\end{gathered}
$$

$w_{D N}$ is the weighting function describing the problem domain and $w_{N}$ is the weighting function describing the region covered by the Neumann boundaries.

The known component of the potential function, $\left(\varphi_{\delta}\right)$ has been constructed fulfilling the Dirichlet - type bound- 
ary conditions along the corona wire and the grounded plate as,

$$
\varphi_{\delta}=\varphi_{0} w_{1} /\left(w_{1}+w_{2}\right)
$$

where $w_{1}$ and $w_{2}$ are the weighting functions describing the grounded plate and corona wire respectively [8] which are obtained as shown in the Figure 2 a.

The unknown component of the potential function, $\left(\varphi_{\delta}\right)$ in accordance with the Ritz method [7], can be approxi-

(a)
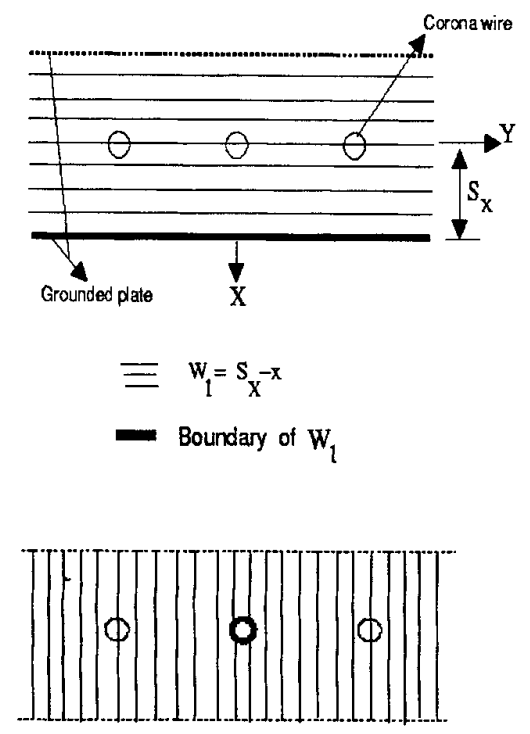

III $w_{2}=\sqrt{\left(x^{2}+y^{2}\right)}-a$

- Boundary of $\mathrm{W}_{2}$

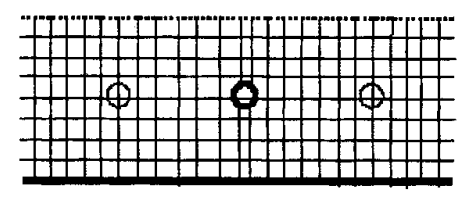

mated by the first $n$ elements of an entire function set as,

$$
\varphi_{\alpha}=\sum F_{k} u_{k}, \quad k=1,2 \ldots, n
$$

where ' $u$ ' is a column vector with unknown coefficients ' $u_{k}$ ' $(k=1,2 \ldots n)$. The $k$ th element of the approximating function $F_{k}$ can be constructed to fulfill the prescribed homogeneous Dirichlet - type boundary conditions as

$$
F_{k}(x, y)=w_{D} f_{k}(x, y), \quad k=1,2, \ldots, n .
$$

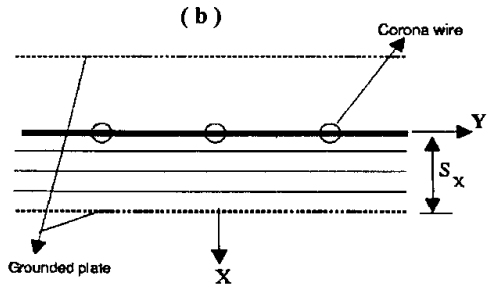

$\equiv \mathrm{w}_{3}=\mathrm{x}$

- Boundary of $\mathrm{w}_{3}$
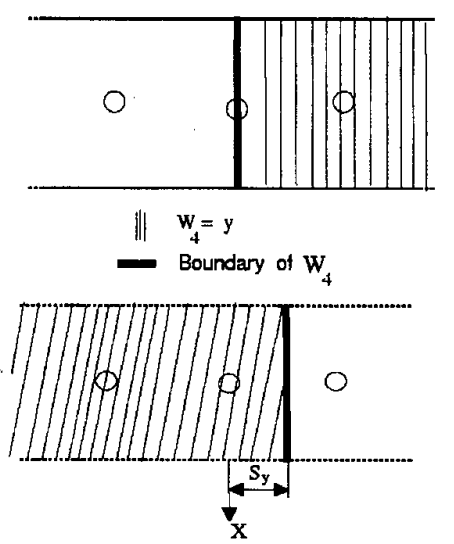

IIII/ $\mathbf{w}_{5}=\mathbf{s}_{\mathbf{y}}-\mathrm{y}$

Boundary of $\mathrm{w}_{5}$

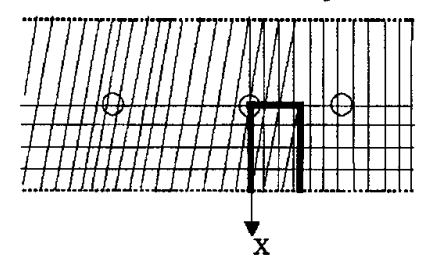

$w_{N}=w_{3} \wedge w_{4} \wedge w_{5}$

Boundary of $\mathrm{W}_{\mathrm{N}}$

(c)

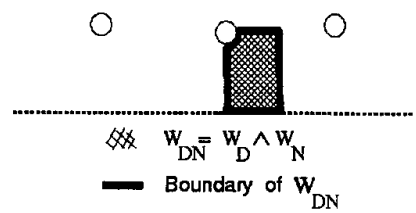

Figure 2. Realization of problem domain using R-functions. (a) Arriving at the function $w_{D}$, (b) Arriving at the function $w_{N}$, (c) Arriving at the function $w_{D N}$ 
where $f_{k}(x, y)$ is the basis function of the approximation in Cartesian coordinates, which has been selected to be the Chebyshev polynomial [9] and $w_{D}$ is the weighting function which has been selected using R-functions describing the region covered by Dirichlet boundaries (Figure 2 a) as,

$$
w_{D}=w_{1} \wedge w_{2}
$$

The weighting function $w_{D}$ has been verified to satisfy the homogenous Dirichlet - type boundary conditions. After determining the approximating function $F_{k}$ the elements of the vector ' $u$ ' must be determined to compute the unknown component of the potential function $\left(\varphi_{\alpha}\right)$. The elements of the vector ' $u$ ' can be determined with the help of the first derivation of Equation (20) which results in a linear set of equations as,

$$
A u=b
$$

where ' $A$ ' is an $n$ th-order quadratic matrix and ' $b$ ' is a column vector with $n$ elements formed from the known component of the potential function and from the space charges. The elements of matrices ' $A$ ' and ' $b$ ' can be determined as

$$
\begin{gathered}
A[k, l]=\int_{\Omega} \epsilon_{0} \nabla F_{k} \cdot \nabla F_{l} d \Omega \\
b[k]=-\int_{\Omega}\left(\epsilon_{0} \nabla \varphi_{\delta} \cdot \nabla F_{k}-\rho F_{k}\right) d \Omega
\end{gathered}
$$

For a given geometry the elements of matrix ' $A$ ' does not change and hence be computed outside the potential loop. After determining the unknown coefficients ' $u_{k}$ ', $\varphi_{\text {ix }}$ can be computed by the Equation (26).

Now for determining the third component in the potential function (equation (22)), $w_{D N}$ must be computed. Figure 2 shows how $w_{D N}$ is obtained using functions $w_{D}$ and $w_{N}$ [8]. From Figure $2 \mathrm{~b}, w_{N}$ is first defined as,

$$
w_{N}=w_{3} \wedge w_{4} \wedge w_{5}
$$

where $w_{3}, w_{4}$ and $w_{5}$ describe the three Neumann boundaries surrounding the problem domain. These functions $w_{3}, w_{4}$ and $w_{5}$ describing the Neumann-boundaries need to be normalized, which is explained in the section $\langle 3.3\rangle$. Then Figure $2 \mathrm{c}$ shows how the problem domain has been realized by the intersection of functions $w_{D}$ and $w_{N}$. The function $w_{D N}$ describing the problem domain is given as,

$$
w_{D N}=w_{D} \wedge w_{N}
$$

The weighting function $w_{D N}$ has been verified to satisfy the Neumann - type boundary conditions along ' $\mathrm{AB}$ ', ' $\mathrm{BC}$ ' and 'AD'. The scalar potential $(\varphi)$ can now be determined from the Equations (22), (23) and (24).

The weighting functions $\left(w_{1}, w_{2}, \ldots\right.$ etc $)$ were determined using R-functions [6]. The section $\langle 3\rangle$ briefly describes the underlying theory of R-functions.

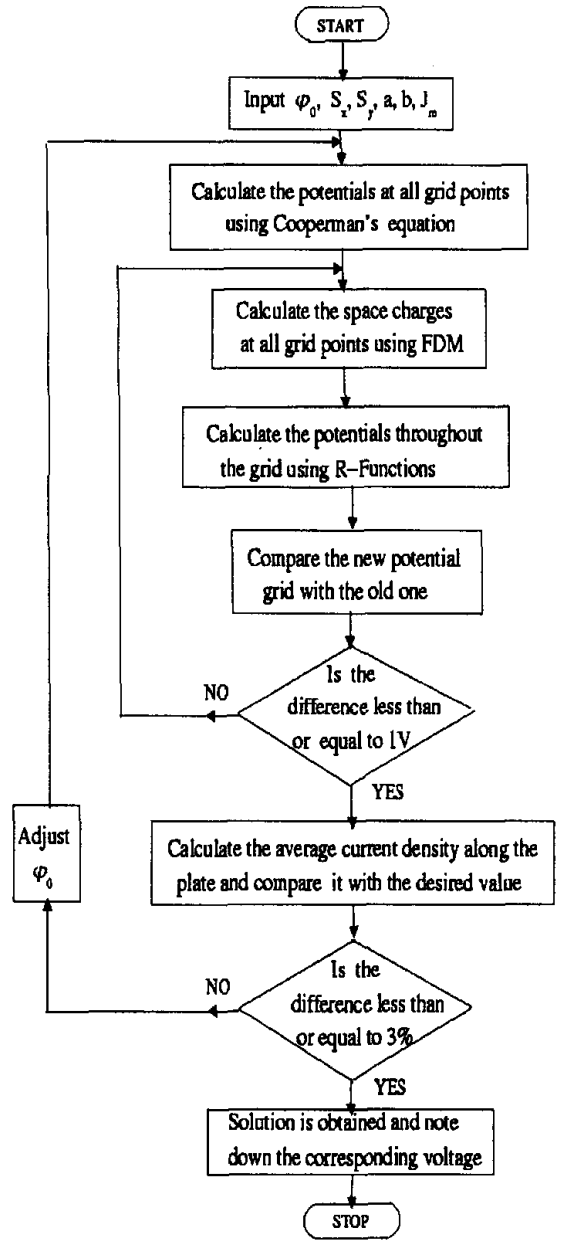

Figure 3. Flow diagram.

\subsection{ALGORITHM AND FLOW DIAGRAM}

Figure 3 shows the flow chart used in the present quasi-analytical technique. An initial value of voltage $\left(\varphi_{0}\right)$ (usually equal to corona onset voltage) was assumed at the wire and the potentials at various grid points have been computed using Cooperman's analytical equation [1]. From the computed potential grid, the space charge grid was generated by solving the current continuity equation. Poisson's equation was then solved by applying the Variational principle using R-functions as a tool. The current continuity equation and the Poisson's equations were solved simultaneously until the difference between two successive potential grids is less than or equal to one volt. The average current density $\left(j_{\text {ave }}\right)$ along the grounded plate can then be computed as,

$$
j_{\mathrm{ave}}=\frac{1}{n} \sum_{j=1}^{n}\left(\rho_{j} b E_{x_{j}}\right)
$$

where $n$ is the number of grid points. The difference between the computed and the desired average current den- 
sities was checked and if the difference was greater than $3 \%$ the voltage at the wire was incremented and the whole procedure was repeated starting from potential grid till convergence is obtained.

\section{UNDERLYING THEORY OF R-FUNCTIONS}

\subsection{DEFINITION}

The $R$-functions were devised by a Russian mathematician V. L. Rvachev [6]. According to this theory, any bounded region can be defined a distance function with respect to the boundary surface.

Figure 4 a shows the region $\Omega$, which is the union of the inner domain $\Omega_{i}$ (shaded portion) and its boundary $\Gamma$,

$$
\text { i.e. } \Omega=\Omega_{i} \cup \Gamma
$$

The inner domain $\left(\Omega_{i}\right)$ and the outer domain $\left(\Omega_{e}\right)$ regions are separated by the boundary $\Gamma$. The R-function ' $w$ ' describe the bounded domain $(\Omega)$ such that it has zero value at any point on the surface $\Gamma$, positive value at any point in the inner domain $\Omega_{i}$ and negative value at any point in the outer domain $\Omega_{e}$.

$$
\begin{aligned}
&>0, \text { if } p \in \Omega_{i} \\
& \text { i.e. } w=0, \text { if } p \in \Gamma \\
&<0, \text { if } p \in \Omega_{e}
\end{aligned}
$$

\subsection{OPERATIONS ON R-FUNCTIONS}

Any region defined by the boundary surfaces can be composed of the union, intersection or complementary of the sub-regions and the logical operations R-conjunction, $\mathrm{R}$-disjunction and R-negation describe them respectively.

The union of the sub-regions is described by the logical operation R-conjunction as shown in Figure $4 \mathrm{~b}$. If $w_{1}$ and $w_{2}$ are $\mathrm{R}$-functions describing the regions $\Omega_{1}$ and $\Omega_{2}$ bounded by the surfaces $\Gamma_{1}$ and $\Gamma_{2}$, then the $R$-function $w$ describing the region $\Omega$ bounded by the surface $\Gamma$ is mathematically formulated on the basis of triangular inequalities as,

$$
w=w_{1} \vee w_{2}=w_{1}+w_{2}+\sqrt{ }\left(w_{1}^{2}+w_{2}^{2}\right)
$$

Similarly, the intersection of the sub-regions is described by the logical operation R-disjunction (Figure 4c) and mathematically formulated as,

$$
w=w_{1} \wedge w_{2}=w_{1}+w_{2}-\sqrt{ }\left(w_{1}^{2}+w_{2}^{2}\right)
$$

Complementary of a region is described by the logical operation R-negation (Figure 4d) and if $w$ is the R-function describing the region $\Omega$ bounded by the surface $\Gamma$, then its complementary region is formulated as,

$$
\text { Negation }(w)=-w
$$

(a)

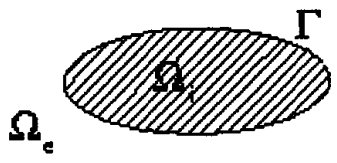

$$
\begin{array}{ll} 
& \text { denotes inner domain }\left(\Omega_{\mathrm{i}}\right) \\
\Omega_{\mathrm{e}} & \text { denotes outer domain }\left(\Omega_{\mathrm{e}}\right) \\
\Gamma \quad \text { denotes boundary of the domain } \\
\text { and } \quad \Omega=\Omega_{\mathrm{i}} \cup \Gamma
\end{array}
$$

(b)

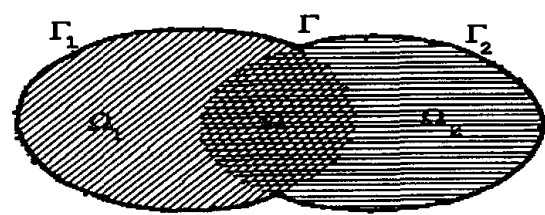

$$
\begin{aligned}
& \text { V } \Omega_{1} \text { domain 些 } \Omega_{2} \text { domain } \\
& \text { entire shaded sres } \mathbf{S} \boldsymbol{\Omega} \text { domsin }
\end{aligned}
$$

Union of sub-regions $\Omega_{1}$ and $\Omega_{2}$

(c)

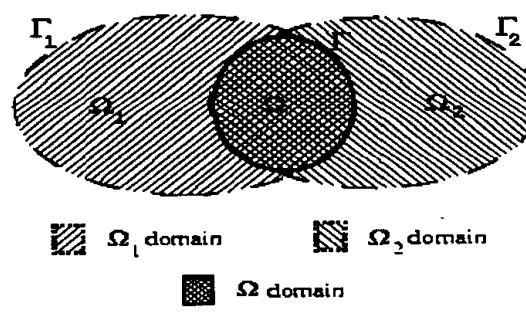

Intersection of sub-regions $\Omega_{1}$ and $\Omega_{2}$

(d)

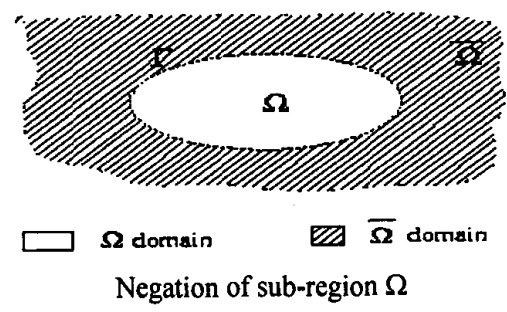

Figure 4. R-functions [7]

(a) Definition; (b) R-Conjunction; (c) R-Disjunction; (d) R-Negation

\subsection{NORMALIZATION PROCESS}

All the functions describing the Neumann-boundaries need to be normalized [6]. If the gradient of the R-function has a non-zero value at any internal point of the region $\Omega$, then the normalized $\mathrm{R}$-function can be defined as

$$
W_{n}=\frac{w}{\left|\nabla_{w}\right|} \text { if }\left.\quad \nabla w\right|_{\Omega} \neq 0
$$


And if the gradient of the $\mathrm{R}$-function has a zero value at any internal point of the region $\Omega$, then the normalized $\mathrm{R}$-function can be defined as

$$
w_{n}=\frac{2}{\sqrt{\left(w^{2}+|\nabla w|^{2}\right)}}=1 \text { if }\left.\nabla w\right|_{\Omega}=0
$$

\subsection{APPLICATIONS}

Combining the global variational method with R-functions, a solution of differential equation can be determined, fulfilling exactly the prescribed boundary conditions in bounded as well as in unbounded case. R-functions can be used to find the solution of mechanical and heating problems. In electromagnetism, R-functions can be used for solving the Poisson, the Helmholtz, the diffusion and the wave equations.

\section{RESULTS AND DISCUSSION}

Performance of an ESP can be determined by its V-I characteristics. To analyze the electrical conditions of a precipitator under various load conditions, V-I characteristics under clean-air conditions are considered as a reference and once these characteristics are obtained, they can be used to diagnose the electrical problems that occur in an existing ESP during its operation.

Figure $5 \mathrm{a}$ shows the validation of the predicted V-I characteristics Penney and Matick's experimental results [10], which are usually considered as reference by many researchers. Our results were also validated with Gene Cooperman's experimental data [11] as shown in Figure 5b. Validation of the inter-electrode potentials was also made with Penney and Matick's results [10] as shown in Figure $5 c$.

Figure 6a shows clearly a better agreement of the results obtained by the proposed quasi-analytical method when compared with the numerical techniques (FDM and FEM) [1,12], for the Penny and Matick's experimental data. Results were also compared with other analytical methods $[11,13]$ as shown in Figure $6 b$.

Figure $7 \mathrm{a}, \mathrm{b}$ and $\mathrm{c}$ shows the variation of the potential, electric field and charge density from wire to plate respectively, for Penney and Matick's ESP geometry [10]. The solid line represents the distribution of the above-mentioned parameters in the absence of the space charges (i.e. Laplacian field). The other three curves show the same parameter variations for various applied voltages and current densities (i.e. with space charges). Higher the potential, higher will be the magnitudes of current densities, field and space charges. It is also seen that there is no change in the shape of these profiles though there is an increase in their magnitudes due to the presence of space charges.

Figure 8a shows the effect of variation of size of the corona wire on the V-I characteristics. It can be observed
( a )

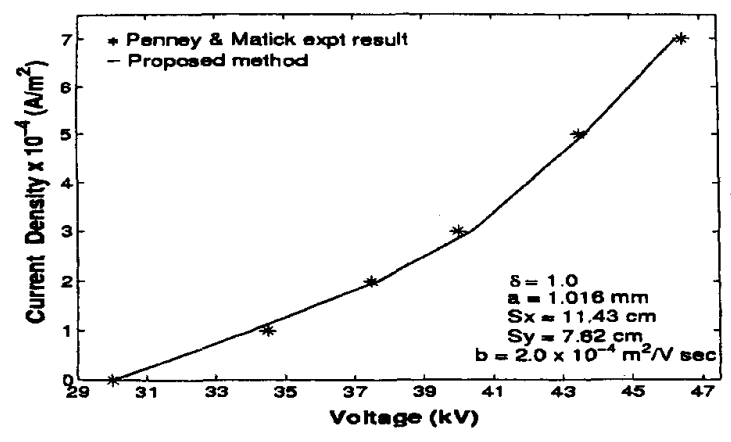

(b)

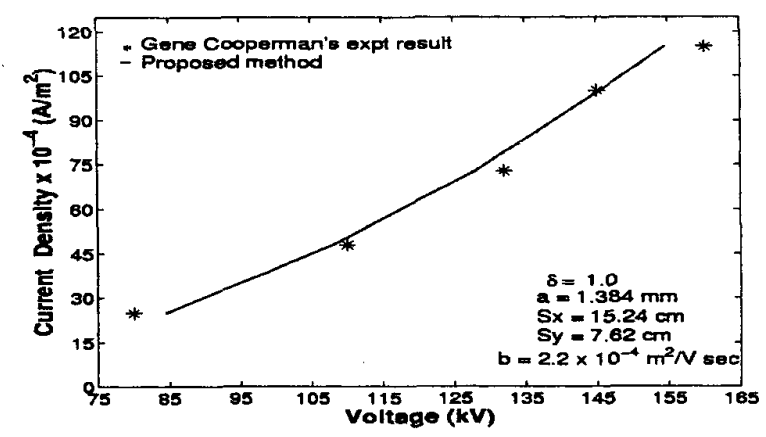

(c)

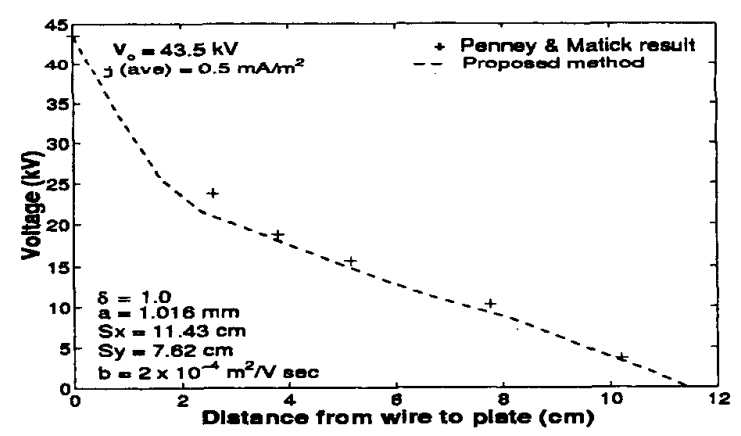

Figure 5. Validation of the predicted results. (a) With Penney \& Matick's expt V-I data [10] (b) With Gene Cooperman's expt V-I data [13]

(c) With Penney \& Matick's inter-electrode potentials [10]

that an increase in the wire size leads to higher corona starting voltage and lower currents for the same applied voltages. The V-I profile is also getting steeper with an increase in the wire size. So where dusts of high resistivity are required to be collected, larger corona wires should be used to give higher fields. Hence while selecting the size of the corona wires the required voltage, electric field and mechanical strength are to be taken into consideration. Figure $8 \mathrm{~b}$ demonstrates the variation of electric field for wire radii of $0.1016,1.016$ and $7 \mathrm{~mm}$ at an average current density of $0.2 \mathrm{~mA} / \mathrm{m}^{2}$. The electric field is found to 
(a)

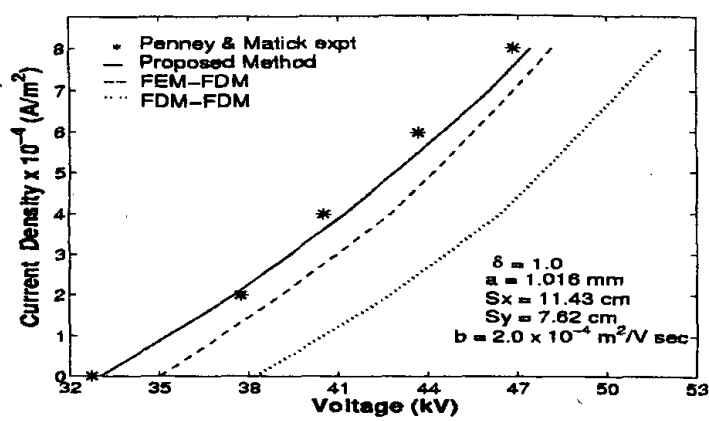

(b)

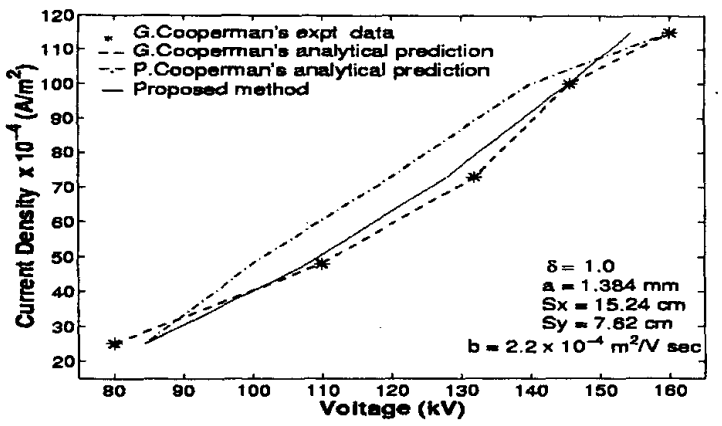

Figure 6. Comparison of the predicted V-I characteristics. (a) With numerical techniques $[1,12]$

(b) With analytical techniques $[6,13]$

increase uniformly with the increase in the radius of the corona wire.

Figure 9a demonstrates effect of wire-plate spacing on the V-I characteristics. It is observed that increasing the wire-plate spacing has only a slight effect on the starting voltage but leads to a larger drop in current for the same applied voltage. Figure $9 \mathrm{~b}$ demonstrates that for the same average current density of $0.5 \mathrm{~mA} / \mathrm{m}^{2}$, the distribution of current along the plate is more uniform for higher wireplate spacing. So configurations with higher wire-plate spacing can be preferred to reduce the non-uniformity in the current distribution.

V-I characteristics were also predicted for a wire of square cross-section of side equal to the diameter of the round wire. Figure 10a demonstrates the comparison of $\mathrm{V}$-I characteristics for both round and square wires. It is observed that for a particular voltage, the current density is slightly low in case of a square-wire as compared to that of a round wire. Figure 10b shows the comparison between the distribution of the current density along the plate (for a 3-wire section) of a round cross-section wire and a square cross-section wire at average current densities of $0.2,0.5$ and $0.7 \mathrm{~mA} / \mathrm{m}^{2}$. It can be observed that unlike the round wire, the current density along the plate (a)

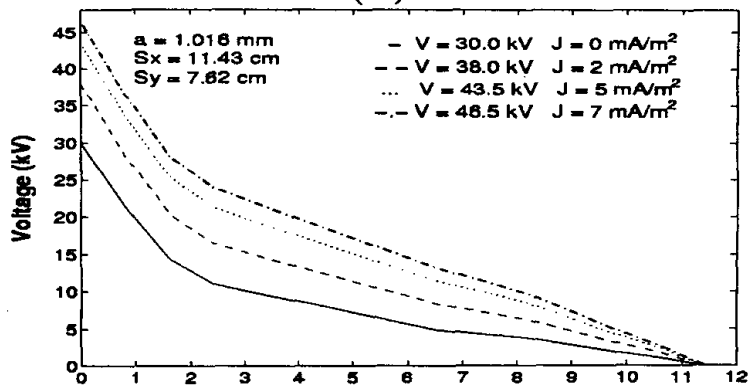

(b)

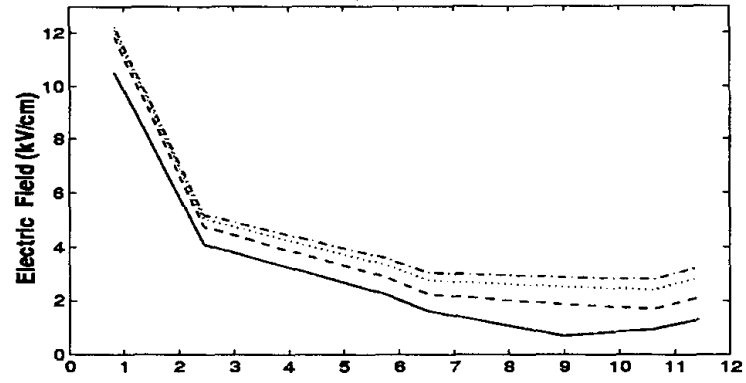

(c)

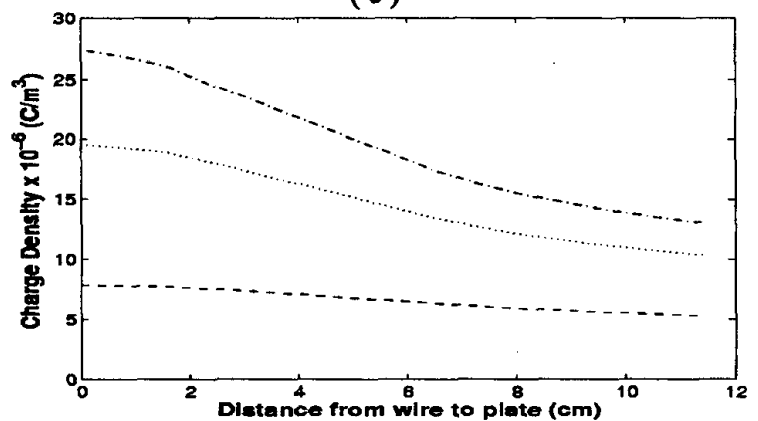

Figure 7. For Penney \& Matick's data.

(a) Variation of potential (V) from wire to plate

(b) Variation of Electric field $(E)$ from wire to plate

(c) Variation of charge density from wire to plate

for square wire cross-section is almost uniform, except at midway between the wires. So a wire of square cross-section can be preferred, where a uniform distribution of the current density is required.

The main advantage of the proposed R-function based method is that electrical characteristics for any practical wire shape or plate shape can be modeled with ease unlike the other conventional numerical techniques. The shape and size of the discharge electrodes are governed by the corona current and mechanical requirements of the system. These corona electrodes typically may be round, square, spiral or barbed in cross-section. The main consideration in case of collection electrodes is to provide stiffness to long plates and to provide some shielding of the 
(a)

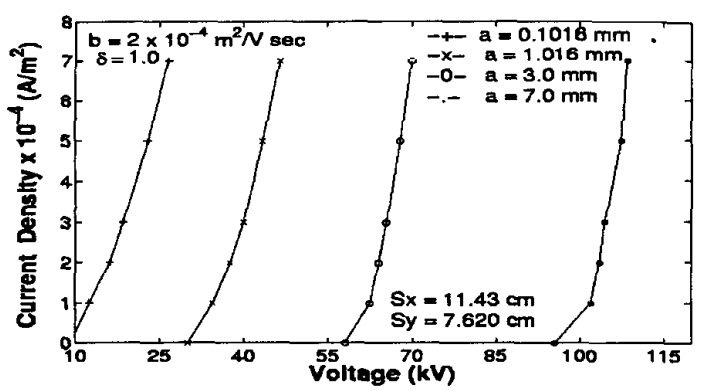

(b)

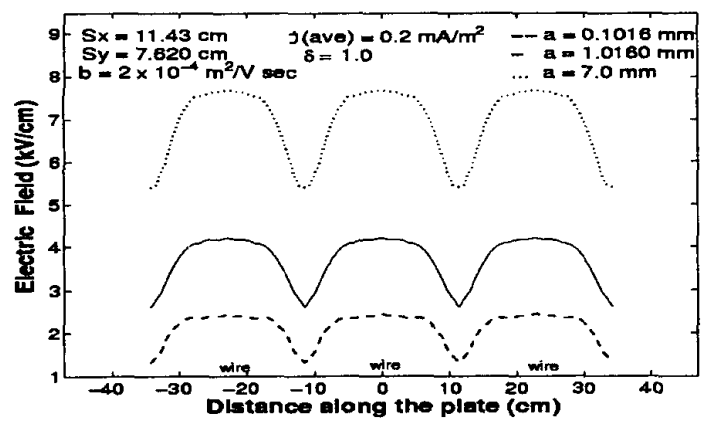

Figure 8. Effect of variation in the corona wire size. (a) On the V-I characteristics

(b) On the electric field (for a 3 wire-section)

(a)

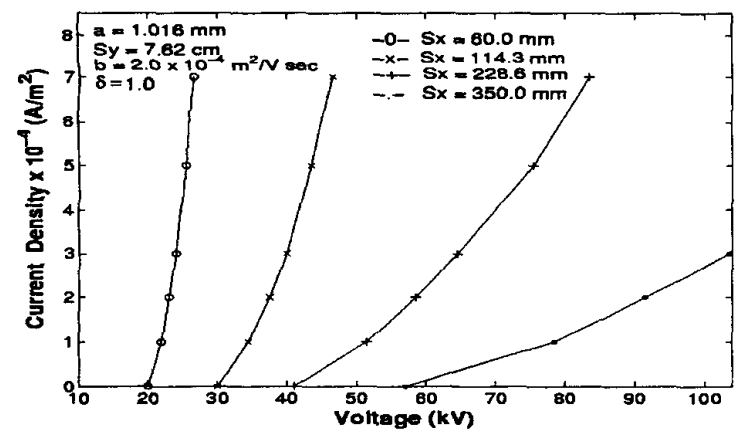

(b)

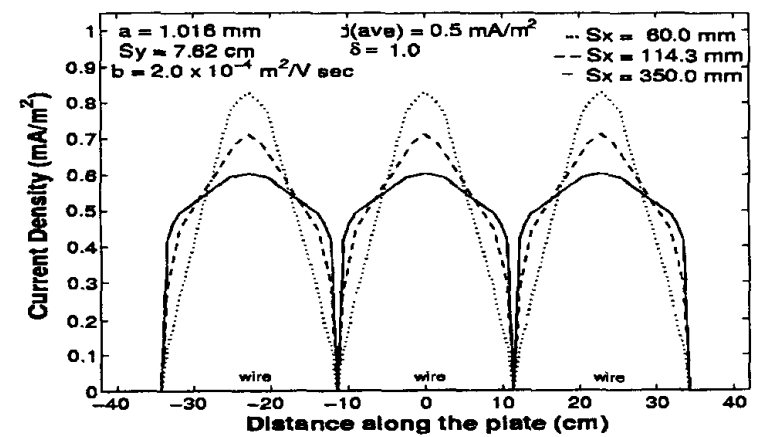

Figure 9. Effect of variation in wire-to-plate spacing. (a) On the V-I characteristics

(b) On the distribution of current density along the plate (a)

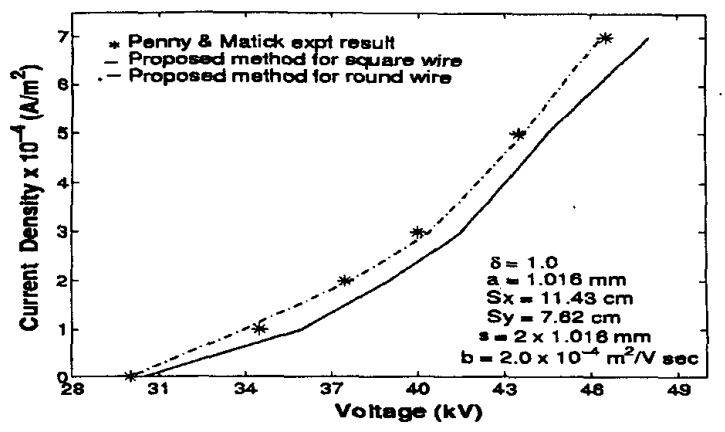

(b)
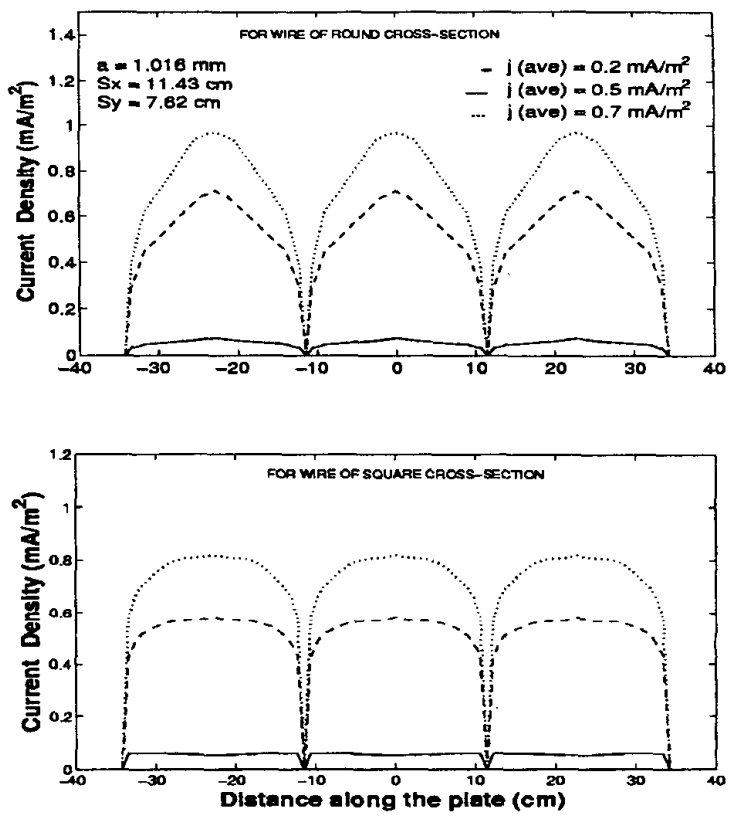

Figure 10. Comparison of round and square cross-section wires. (a) V-I characteristics; (b) Current density distribution along the grounded plate ( 3 wire-section) for round and square wires.

collecting dust layer. These grounded plates may be flat, zig-zag, offset, V-plates or barbed plates [14]. Modeling the different shapes of the electrodes in an ESP will be much easier with R-functions (though we have no literature supporting this at present) than with any other techniques. The proposed method has also better accuracy when compared to existing numerical techniques (FDM and FEM) as is obvious from Figure 6a. This proposed method is limited to only for DC energized precipitators. Only the dust free V-I characteristics can be modeled with the proposed method.

\section{CONCLUSIONS}

$A$ new quasi-analytical approach based on combined $\mathrm{A}_{\text {finite difference and } \mathrm{R} \text {-functions has been proposed }}$ for the prediction of V-I characteristics in a duct-type pre- 
cipitator. The main advantage of this approach is that any shape of the corona electrodes can be modeled with ease and hence, helps in designing a precipitator with new innovative shapes of the corona and grounded electrodes. The predictions of the proposed method showed a very good accuracy with the experimental results when compared with other numerical techniques. The method can also be used to consider the bends in the grounded plate also, which otherwise is difficult to simulate with other existing numerical techniques.

\section{REFERENCES}

[1] J. R. McDonald, W. B. Smith, H. W. Spencer III and L. E. Sparks, "A Mathematical Model for Calculating Electrical Conditions in Wire-Duct Precipitator", J. Appl. Phys., Vol. 48, pp. 2231-2243, 1977

[2] A. J. Medlin, Electro Hydrodynamic Modeling of Fine Particle Collection in Electrostatic Precipitators, PhD Thesis, School of Physics, University of New South Wales, May 1998.

[3] T. Barbarics, H. Igarashi, A. Ivangi and T. Honma, "Electrostatic Field Calculation Using R-functions and the Method of Characteristics in Electrostatic Precipitator", J. Electrostatics, Vol. 38, pp. 269-282, 1997.

[4] F. W. Peek, Jr., Dielectric phenomena in High Voltage Engineering, McGraw-Hill, 1929.

[5] N. Thirumaran, A Modified Approach to Predict Voltage-Current Characteristics of an Electrostatic Precipitator, MSc(Engg)
Thesis, Dept. of High Voltage Engineering, Indian Institute of Science, Bangalore, India, 2000.

[6] A. Ivanyi, R-functions in Electromagnetism, Technical Report, TUB-TR-93-EE08, Budapest, 1993.

[7] L. V. Kantorovich and V. J. Krylov, Approximate Methods of Higher Analysis, Wiley, New York, NY, 1964.

[8] B. S. Rajanikanth and D. V. S. Sarma, Modeling the Prebreakdown Characteristics of a Duct-type Precipitator Based on FDM and Variational Principle Using R-functions as a Tool, 12th International Symposium on High Voltage Engineering, India, 2001.

[9] R. W. Hamming, Introduction to Applied Numerical Analysis, McGraw-Hill, New York, 1971.

[10] G. W. Penney and R. E. Matick, "Potentials in DC corona fields", Trans. AIEE, Part-I, Vol. 79, pp. 91-99, 1960

[11] G. Cooperman, "A new Voltage-Current Relation for Duct Precipitators Valid for Low and High Current Densities," IEEE Trans. on IAS, Vol. 17, pp. 236-239, 1981.

[12] Gregory A. Kallio and David E. Stock, "Computation of Electrical Conditions Inside Wire-Duct Electrostatic Precipitators Using a Combined Finite-Element, Finite-Difference Technique," J. Appl. Phys., Vol. 59, pp. 1799-1806, 1986.

[13] P. Cooperman, "A Theory of Space Charge Limited Currents With Applications to Electrostatic Precipitator", Trans. AIEE, Part I, pp. 47-50, 1960.

[14] S. Oglesby, Jr. and G. B. Nichols, Electrostatic Precipitation, Marcel Dekker Inc., NY, USA, 1978.

Manuscript received on 21 August 2001, in final form 10 October 2001. 